\title{
ON THE REPRESENTATION OF NUMBERS MODULO $m^{*}$
}

\author{
BY E. D. RAINVILLE
}

Dirichlet and Kronecker $\dagger$ extended the notion of primitive root to the case of any composite modulus. The classical Kronecker-Dirichlet theorem may be stated as follows. Let $m=2^{\alpha_{0}} p_{1}{ }^{\alpha_{1}} \cdots p_{v}{ }^{\alpha_{v}}$, where the $p^{\prime}$ 's are distinct odd primes. Determine $g_{k}$, a primitive root of $p_{k} \alpha_{k}$, for $k=1,2, \cdots, v$. Form

$$
\lambda_{k}=g_{k}+p_{k}{ }^{\alpha} \beta_{k} \equiv 1 \bmod m / p_{k}{ }^{\alpha k},
$$

and, if $\alpha_{0}>1$,

$$
\begin{aligned}
\lambda & =-1+2^{\alpha_{0}} \beta \equiv 1 \quad \bmod m / 2^{\alpha_{0}}, \\
\lambda_{0} & =5+2^{\alpha_{0}} \beta_{0} \equiv 1 \quad \bmod m / 2^{\alpha_{0}} .
\end{aligned}
$$

Then, for $(n, m)=1, n$ is uniquely represented modulo $m$ by

$$
n \equiv \lambda^{i} \lambda_{0}{ }^{i_{0}} \prod_{k=1}^{v} \lambda_{k}{ }^{i_{k}} \bmod m
$$

where the exponents are restricted by the inequalities

$0 \leqq i \leqq 1, \quad 0 \leqq i_{0} \leqq \phi\left(2^{\alpha_{0}-1}\right)-1, \quad 0 \leqq i_{k} \leqq \phi\left(p_{k}^{\alpha k}\right)-1$.

If $\alpha_{0} \leqq 1, \lambda$ and $\lambda_{0}$ are not to be formed, hence $i=i_{0}=0$ automatically.

In the course of another investigation a further extension to the case of general $n$ (dropping the restriction $(n, m)=1$ ) became necessary. This is the object of the present note.

TheOREM. Let $m=2^{\alpha_{0}} p_{1} \alpha_{1} \cdots p_{v}{ }^{\alpha_{v}}$ (p's distinct odd primes). Determine $g_{k}$, a primitive root $\$$ of $p_{k}{ }^{2}, k=1,2, \cdots, v$. Form

$$
\lambda_{k}=g_{k}+p_{k}^{\alpha_{k}} \beta_{k} \equiv 1 \quad \bmod m / p_{k}^{\alpha_{k}}
$$

and, if $\alpha_{0}>1$,

* Presented to the Society, March 18, 1933.

$\dagger$ Dickson, History of the Theory of Numbers, vol. 1, pp. 185, 192.

$\ddagger$ The root $g_{k}$ is then also a primitive root of $p_{k}^{n}, n>0$ (Dirichlet-Dedekind, Zahlentheorie, 4th ed., 1894, p. 334). 


$$
\begin{aligned}
\lambda & =-1+2^{\alpha_{0}} \beta \equiv 1 \quad \bmod m / 2^{\alpha_{0}}, \\
\lambda_{0} & =5+2^{\alpha_{0}} \beta_{0} \equiv 1 \quad \bmod m / 2^{\alpha_{0}} .
\end{aligned}
$$

Then any $n$ is uniquely represented modulo $m$ by

$$
n \equiv 2^{\sigma_{0}} \lambda^{i} \lambda_{0}^{i_{0}} \prod_{k=1}^{v} p_{k}^{\sigma_{k} \lambda_{k}}{ }_{k}^{i_{k}} \bmod m
$$

where

$$
0 \leqq \sigma_{0} \leqq \alpha_{0}, \quad 0 \leqq \sigma_{k} \leqq \alpha_{k}, \quad(k=1,2, \cdots, v),
$$

and the other exponents are subject to the restrictions that

if $\sigma_{0} \geqq \alpha_{0}-1$, then $i=i_{0}=0$;

if $0 \leqq \sigma_{0} \leqq \alpha_{0}-2$, then $0 \leqq i \leqq 1$ and $0 \leqq i_{0} \leqq \phi\left(2^{\alpha_{0}-\sigma_{0}-1}\right)-1$;

if $0 \leqq \sigma_{k} \leqq \alpha_{k}$, then $0 \leqq i_{k} \leqq \phi\left(p_{k}{ }^{\alpha}{ }^{-\sigma} k\right)-1$,

for $k=1,2, \cdots, v$.

PROOF. In order to show that all numbers are represented uniquely by (A) we prove (1) that the number of such representations is $m$, and (2) that no two representations are congruent modulo $m$.

(1) The number of combinations of exponents $\sigma_{0}, i, i_{0}$ due to letting $\sigma_{0}$ assume all permissible values is evidently

$$
\begin{aligned}
1+1+2 \cdot \sum_{\sigma_{0}=0}^{\alpha_{0}-2} \phi\left(2^{\alpha_{0}-\sigma_{0}-1}\right) & =1+\phi(2)+\sum_{\sigma_{0}=0}^{\alpha_{0}-2} \phi\left(2^{\alpha_{0}-\sigma_{0}}\right) \\
& =\sum_{\sigma_{0}=0}^{\alpha_{0}} \phi\left(2^{\alpha_{0}-\sigma_{0}}\right)=2^{\alpha_{0}} .
\end{aligned}
$$

Similarly, for any $k=1,2, \cdots, v$, the number of combinations of exponents $\sigma_{k}, \alpha_{k}$ due to letting $\sigma_{k}$ assume all permissible values is

$$
\sum_{\sigma_{k}=0}^{\alpha_{k}} \phi\left(p_{k}^{\alpha_{k}-\sigma_{k}}\right)=p_{k}^{\alpha_{k}} .
$$

Hence, combining these results, we have for $T$, the total number of representations,

$$
T=2^{\alpha_{0}} p_{1}^{\alpha_{1}} \cdots p_{v}^{\alpha_{v}}=m .
$$


(2) The uniqueness is made to depend upon the KroneckerDirichlet theorem in the following manner. Suppose, with the restrictions of our theorem, that

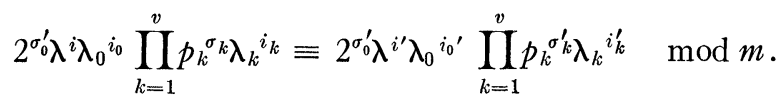

Then, since $\lambda, \lambda_{0}$ and $\lambda_{k}$ are relatively prime to $m$,

$$
\sigma_{0}=\sigma_{0}^{\prime}, \quad \sigma_{k}=\sigma_{k}^{\prime}, \quad(k=1,2, \cdots, v),
$$

and we have

(B) $\quad \lambda^{i} \lambda_{0} i_{0} \prod_{k=1}^{v} \lambda_{k}{ }^{i k} \equiv \lambda^{i^{\prime}} \lambda_{0}{ }^{i_{0}} \prod_{k=1}^{v} \lambda_{k}{ }^{i k^{\prime}} \bmod 2^{\alpha_{0}-\sigma_{0}} \prod_{k=1}^{v} p_{k}^{\alpha_{k}-\sigma_{k}}$

Since $\lambda_{k}$ is a primitive root of $p_{k}^{2}$, it is a primitive root of $p_{k} \alpha_{k}-\sigma_{k}$. From the restrictions of the theorem, we conclude that $0 \leqq i_{k}, i_{k}^{\prime} \leqq \phi\left(p_{k} \alpha_{k}-\sigma_{k}\right)-1$. Further, $0 \leqq i_{0}, i_{0}^{\prime} \leqq \phi\left(2^{\alpha_{0}-\sigma_{0}-1}\right)-1$, if only $\alpha_{0}-\sigma_{0}>1$. Again, if $\alpha_{0}-\sigma_{0}>1$, we know that $0 \leqq i, i^{\prime} \leqq 1$.

Thus all conditions of the Kronecker-Dirichlet theorem are satisfied in (B) for the modulus

$$
2^{\alpha_{0}-\sigma_{0}} \prod_{k=1}^{v} p_{k}^{\alpha_{k}-\sigma_{k}}=\left(m / 2^{\sigma_{0}}\right) \prod_{k=1}^{v} p_{k}{ }^{\sigma k},
$$

and the representation $\lambda^{2} \lambda_{0} i_{0} \prod_{k=1}^{v} \lambda_{k} i_{k}$ is a unique representation modulo $\left(m / 2^{\sigma_{0}}\right) \prod_{k=1}^{v} p_{k}^{\sigma_{k}}$, and, a fortiori, modulo $m$. Therefore, the representation (A) is unique modulo $m$.

The University of Colorado 